\title{
Sitzung vom 12. October 1896.
}

Vorsitzender: Hr. H. La n d o lt, Präsident.

Das Protokoll der letzten Sitzung wird genebmigt.

Der Vorsitzende begrüsst das auswärtige Mitglied Hrn. Dr. C. Mangold aus Wien, sowie den General-Secretär der Gesellschaft, Hrn. Prof. P. Jacobson, welcher der Sitzung zum ersten Male als einheimisches Mitglied wieder beiwohnt.

Zu ausserordentlichen Mitgliedern werden verkündet die Herren:

$\left.\begin{array}{l}\text { Illig, Robert, } \\ \text { Kopp, Otto, }\end{array}\right\}$ Giessen;

Grunwald, Emil, ?

Günther, Adolf, $\}$ Berlin;

Tyrer, Thomas, London;

Muthmañ, Prof. Dr. W., München;

Casares, Prof. Dr. José, Barcelona.

Herren:

Zu ausserordentlichen Mityliedern werden vorgeschlagen die

Gray, Thomas, Technical College, Glasgow, 204 George-Street

Kellas, Alexander, University College, London

Lehnert, Dr. H., Wachwitz bei Dresden

(durch L. Gattermann und K. A uwers);

Osius, F. W., Ludwigstr. 24, Giessen (durch H. Finger und K. Elbs);

Houben, Josef, Breitestr. 106, Bonn) (durch Jagelki, Wilhelm, Markt 35, Bonn R. Anschütz Sears, Arthur, 178 Devonshire-Street, Boston, Mass., U. S. A.

und

J. Bredt);

Bailey, James R., Austin, Texas, U. S. A. (durch J. Thiele und G. Bender);

Schwalbe, Dr. Carl, Badische Anilin- und Sodafabrik in Ludwigshafen a. Rh. (durch C. Schraube und G. Körner.) 
Für die Bibliothek sind als Geschenke eingegangen:

774. Otto, Rob. Anleitung zur Ausmittelung der Gifte. T. Aufl. 2. Hălfte. Braunschweig 1896.

773. Sammlung chemischer und chemisch-technischer Vorträge. Hrggbn. von Felix B. Abrens. I. Band. Heft 6: Die Einführung einheitlicher Analysenmethoden von Hans Freiherrn von Jüptner. Heft 7-8: Die Abwässer der Fabriken von Hans Benedikt. Stuttgart 1896. 789. Mangold, Carl. Die Stearinindustrie. Weimar 1896.

790. Festschrift der Naturforschenden Gesellschaft in Zürich 1746 bis 1896. 2 Thle. Zürich 1896.

Der Vorsitzende:

H. Landolt.

Der Schriftführer:

A. Pinner.

\section{Mittheilungen.}

\section{Carl Bülow: Oeber das Verhalten des o-Nitro-p-phenylendiamins gegen salpetrige Säure.}

[Erste vorläufige Mittheilung aus dem chemischen Laboratorium der Universität Tübingen.]

(Eingegangen am 10. August; mitgetheilt in der Sitzung von Hrn. Harries.)

Ladenburg ${ }^{1}$ ) erbielt durch Einwirkung von Nitrit auf eine schwefelsaure Lösung von $o$-Nitro-p-phenylendiamin schon iu der Kälte ein Lraunes amorphes Pulver, welches indessen nicht näber untersucht wurde, da es iha wichtiger erschien, den äbnlichen aus $p$-Phenylendiamin entstehenden Körper $z \mathrm{u}$ isoliren.

Beschäftigt mit der Einwirkung von Nitrit auf Diamine der verschiedensten Art, habe ich das Ladenburg'sche Mononitro-p-phenylendiamin bergestellt. Es liefert bei der Behandlung mit einem grossen Ueberschuss von wässriger Salzsäure keine zweisäurigen, sondern nur einsäurige Salze - Ber. für $\mathrm{NO}_{2} \cdot \mathrm{C}_{6} \mathrm{H}_{3} \cdot\left(\mathrm{NH}_{2}\right)_{2} \cdot \mathrm{HCl}$ : $\mathrm{Cl} 18.70 \mathrm{pCt}$; gefunden aus $0.0975 \mathrm{~g}$ Substanz: $\mathrm{AgCl} 0.074 \mathrm{~g}=$ Cl $18.77 \mathrm{pCt}$. - und gleicht in dieser Beziehuvg also rollkommen dem Gottlieb'schen $p$-Nitro-o-Phenylendiamin. Hier wie dort ist eine Erklärung der Veränderuug der Sättigungscapacität, gegenüber den nicht nitrirten Basen, nur in dem Vorbandensein der Nitrogruppe zu suchen, welche in hervorragender Weise die Basicität aromatischer Amine alschwächt. Mononitranilin z. B. ist eine sebr schwache

1) Diese Berichte $17,147$. 